\title{
REPUTAÇÃO CORPORATIVA: O DESAFIO DA ANÁLISE
}

\author{
Marta F. S. Carvalho* \\ António Carrizo Moreira ${ }^{\dagger}$
}

\begin{abstract}
RESUMO: Embora a reputação corporativa tem sido objeto de estudo desde há algumas décadas, só a partir dos anos 90 é que o interesse pela temática se intensificou. Com a competitividade de mercado as empresas precisam de todos os ativos que lhes possam acrescentar valor, sendo a reputação um deles. Assim, foi decidido fazer uma revisão à literatura que explana os modelos de avaliação da reputação e a influência dos principais stakeholders. Dada a complexidade do tema, foi igualmente decidido complementar a análise dos modelos com uma revisão das principais variáveis/constructos que se relacionam com a reputação corporativa.
\end{abstract}

Palavras-chave: reputação corporativa, identidade, imagem

Abstract: Although corporate reputation has been studied for several decades, its interest has intensified only from the 1990s onwards. With the growing market competitiveness firms need to take advantage of their full assets in order to add value to their customers, and the reputation is one of them. Accordingly, it was decided to review the literature that analyses reputation models and the influence of key stakeholders. Given the complexity of the issue, it was also decided to complement the analysis of models with a review of key variables / constructs that influence corporate reputation.

Keywords: corporate reputation, identity. image.

Docente do Instituto superior da Maia, ISMAI. Doutoranda em Marketing e Estratégia na Universidade de Aveiro.

${ }^{\dagger}$ Professor Auxiliar no DEGEI, Universidade de Aveiro. 


\section{INTRODUÇÃO}

A tarefa de descortinar o conceito de reputação revela-se árdua, sobretudo por esta estar incluída em diferentes áreas de saber, que o entendem de forma distinta, e pela existência de diferentes escolas de pensamento, com um entendimento próprio no que concerne aos stakeholders que devem ser tidos em consideração no seu estudo.

Independentemente da escola ou da área de saber, é fulcral perceber que existe uma relação positiva entre reputação e desempenho organizacional, devendo esta relação ser trabalhada. De facto, muita importância tem sido dada à marca; no entanto a reputação também dependente de fatores como identidade e imagem, são os seus elementoschave (Balmer \& Soenen, 1999).

Para lidar com a identidade é necessário tem em consideração os stakeholders internos pois têm um papel preponderante na projeção dessa identidade junto dos stakeholders externos. Assim, é necessário uma estratégia de gestão da identidade. Como as perceções dos públicos externos são influenciadas pelas perceções dos funcionários (Dortok, 2006), a imagem será, em parte, afetada por esta influência do stakeholder interno, o que se reflete na reputação.

No presente artigo pretende-se estudar a temática reputação corporativa, mais especificamente perceber o seu conceito e elementos associados, bem como os instrumentos de avaliação da reputação. Para tal, procedeu-se a uma revisão de literatura nas áreas da gestão, comunicação e marketing.

Assim, começar-se-á por apresentar as diferentes abordagens ao conceito de reputação, segundo diferentes áreas de saber e diferentes autores. De seguida analisa-se em detalhe os conceitos inerentes à reputação: identidade e imagem, incluindo-se o estudo do papel da comunicação corporativa. No ponto seguinte apresenta-se o resultado da investigação a propósito da avaliação da reputação corporativa. Para terminar, inferem-se as principais conclusões e limitações.

\section{O CONCEITO}

Considerando a competitividade do atual mundo empresarial, a reputação corporativa apresenta-se como um dos principais ativos intangíveis das empresas na obtenção de vantagem competitiva em relação aos seus concorrentes. Na busca do conceito, não se encontra uma 
opinião unânime, chegando a ser entendida como sinónimo de imagem corporativa (Caruana, 1997).

As definições do conceito variam em função do autor e da corrente que seguem, colocando o enfoque em diferentes stakeholders. Chun (2005) menciona a existência de três escolas de pensamento que encaram a reputação de acordo com um princípio característico: Relacional, Avaliativa e Impressionista. O quadro I sintetiza o pensamento de cada uma delas.

\section{Quadro I}

Reputação: Três escolas do pensamento

\begin{tabular}{|l|l|}
\hline Escola & Características \\
\hline \multirow{3}{*}{ Relacional } & $\begin{array}{l}\text { Foco nos stakeholders internos e externos; Reputação é reflexo } \\
\text { da visão interna (identidade) e externa (imagem) da } \\
\text { organização; Múltiplos stakeholders. }\end{array}$ \\
\hline Avaliativa & $\begin{array}{l}\text { Avaliada pelo seu valor financeiro; Preocupada com a } \\
\text { performance; Um stakeholder (investidor ou gestor). }\end{array}$ \\
\hline \multirow{3}{*}{ Impressionista } & $\begin{array}{l}\text { Avaliada em termos da perceção dos stakeholders; Termos } \\
\text { usados: imagem, identidade e personalidade; Centrada } \\
\text { essencialmente num stakeholder (consumidor ou funcionário). }\end{array}$ \\
\hline
\end{tabular}

Fonte: Elaborado a partir de Chung (2005)

Claramente, cada escola encara a reputação de uma forma particular. No que concerne ao número de stakeholders verifica-se que as escolas avaliativa e impressionista estão mais preocupadas com os interesses de um stakeholder individual, considerando as suas características e interesses particulares, entendendo a reputação como o reflexo das perceções dos diferentes stakeholders a propósito da organização.

De facto, no que concerne ao funcionário enquanto agente neste processo, Dortok (2006) afirma que o funcionário desempenha um papel importante no processo de formação da sua reputação e alcance de uma melhor posição no mercado. $\mathrm{O}$ autor considera que existe uma troca recíproca, onde as empresas que têm boa reputação atraem bons funcionários que por seu lado terão um bom desempenho, servindo melhor o cliente. Assim, a perceção de outros stakeholders é influenciada pela perceção dos funcionários, sobre a sua própria organização.

A escola avaliativa entende a reputação sob o ponto de vista financeiro. Ela tem as suas raízes na estratégia e economia, preocupandose com a performance, procurando auscultar a satisfação dos investidores (Chun, 2005). No entendimento da escola relacional, vários stakeholders devem ser considerados, e não apenas o funcionário ou investidor, pois a reputação será o resultado da perceção da identidade, através do 
funcionário, e da perceção da imagem, através dos diferentes stakeholders externos.

A riqueza da multidisciplinaridade do conceito de reputação está bem expresso no trabalho Smaiziene e Jucevicius (2009), conforme se apresenta no quadro II, onde são apresentadas diversas perspetivas.

\section{Quadro II}

Entendimento da reputação de acordo com diferentes disciplinas

\begin{tabular}{|l|l|}
\hline \multicolumn{1}{|c|}{ Disciplina } & \multicolumn{1}{c|}{ Interpretação do conceito de reputação } \\
\hline Sociologia & $\begin{array}{l}\text { Entendida como um fenómeno social e característica da sociedade } \\
\text { moderna. }\end{array}$ \\
\hline Psicologia & $\begin{array}{l}\text { É geralmente analisada a um nível individual. A reputação corporativa } \\
\text { habilita a organização a prever o futuro da organização relativamente } \\
\text { aos diferentes stakeholders. }\end{array}$ \\
\hline Economia & Considerada como um sinal sobre as presumíveis ações no mercado. \\
\hline Marketing & $\begin{array}{l}\text { Não é vista como o resultado das ações de marketing. É tratada como } \\
\text { uma das forças do ambiente organizacional. }\end{array}$ \\
\hline $\begin{array}{l}\text { Gestão } \\
\text { impressionista }\end{array}$ & $\begin{array}{l}\text { É definida sob o ponto de vista do impacto que os diferentes eventos } \\
\text { podem ter nos níveis de confiança. }\end{array}$ \\
\hline Estratégia & $\begin{array}{l}\text { Encarada como um ativo intangível que tem impacto na } \\
\text { competitividade da organização, que lhe permitirá competir com mais } \\
\text { forças no mercado. }\end{array}$ \\
\hline $\begin{array}{l}\text { Relaçães } \\
\text { Públicas }\end{array}$ & $\begin{array}{l}\text { Tratada como prática e objeto da disciplina. No entanto, verifica-se que } \\
\text { as diferentes ações para com os diferentes stakeholders serão } \\
\text { instrumentos que contribuirão para a projeção da imagem que terá } \\
\text { posteriormente o seu impacto na reputação. }\end{array}$ \\
\hline $\begin{array}{l}\text { Recursos } \\
\text { Humanos }\end{array}$ & $\begin{array}{l}\text { Centrada nos stakeholders internos (funcionários), perceciona-os como } \\
\text { embaixadores da reputação. Estes serão os grandes responsáveis pela } \\
\text { representação da organização aos olhos dos restantes stakeholders. }\end{array}$ \\
\hline
\end{tabular}

Fonte: Elaborado a partir de Smaiziene e Jucevicius (2009)

Pela análise do quadro II é possível inferir que são vários os inputs trazidos pelas diferentes disciplinas para o conceito de reputação. De uma forma ou de outra todas se centram no alcance da competitividade da organização, ainda que através de diferentes stakeholders, o que também dificulta o consenso a propósito do conceito.

Barnett, Jerminer e Lafferty (2006) reiteram essa multiplicidade de interpretações tendo identificado três grandes grupos com significados diferentes a propósito de reputação. No primeiro grupo a reputação é entendida enquanto "consciência/identificação" com a organização. Os stakeholders sentem-se ligados à organização através de um conjunto de perceções, e mesmo de emoções, não fazendo juízos de valor a propósito da organização. No segundo grupo, ela é vista como "avaliação", pois os stakeholders são vistos como elementos que fazem julgamentos e 
avaliações a propósito do desempenho e comportamento da organização. No terceiro grupo, a reputação é encarada como "qualidade", pois é considerada, sob o ponto de vista do valor que traz para a organização, como um recurso intangível, económico (Barnett, Jerminer \& Lafferty, 2006).

Pelo exposto até ao momento, pode-se afirmar que o entendimento da reputação é multifacetado, dado que envolve perceções e valores, inferidos pelos diferentes stakeholders da organização. no entanto convém esclarecer que especificamente associados ao conceito de reputação estão outros elementos chave como: identidade e imagem organizacional.

\section{IDENTIDADE, IMAGEM E REPUTAÇÃO}

Como apresentado, a reputação resulta das perceções e da avaliação feita pelos stakeholders. Assim, para que estes procedam a essa avaliação será necessário entender como os conceitos de identidade e imagem conduzem a essa avaliação.

A identidade da organização é considerada a realidade da organização, i. e., como se vê a si própria. Traduz a sua personalidade ancorada no comportamento dos seus membros (Chun, 2005; Brady, 2005). Está intrinsecamente relacionada com os sentimentos do funcionários relativamente à organização, sendo portadora de um conjunto de símbolos que a caracterizam (Barnett, Jerminer \& Lafferty, 2006; Walker, 2010).

Gray e Balmer (1998) apontam que identidade como resultado das seguintes quatro componentes: a) estratégia, que engloba a forma como a empresa está no mercado e os objetivos da organização; b) filosofia, onde se definem os valores e crenças da organização a nível dos gestores de topo; c) cultura, através da qual se efetua a difusão dos valores, crenças para toda a organização, que reflete a filosofia; e d) design organizacional, que traduz a forma de organização e estruturação da empresa.

A gestão da identidade (Balmer e Soenen, 1999) envolve a comunicação da visão dos fundadores/gestores da organização. Esta visão estratégica é traduzida por manifestações comportamentais, visuais e verbais que, por sua vez, conduzem a uma imagem positiva da organização.

Esta imagem representa uma idealização que os diferentes públicos têm da organização, que é um reflexo da identidade. Ela corresponde à 
avaliação que os stakeholders fazem da organização. A imagem é construída através de um conjunto de esforços comunicativos coordenados, onde se incluem o nome, o logótipo, a sinalética da organização, bem como a publicidade corporativa (Gray e Balmer, 1998; Walker, 2010). Barnett, Jerminer e Lafferty (2006) referem que a identidade se reflete na imagem através da comunicação, de ações de relações públicas e de marketing.

Enquanto a imagem se refere à ideia mental que os públicos têm da organização, a reputação envolverá os juízos de valor que esses públicos fazem sobre os atributos da organização (Barnett, Jerminer \& Lafferty, 2006; Gray \& Balmer, 1998; Walker, 2010). Brady (2005) acrescenta que a reputação engloba as múltiplas imagens individuais, representando os juízos cumulativos dos públicos ao longo do tempo. Neste sentido percebe-se que se a imagem se pode construir de forma relativamente rápida, a reputação demora mais tempo para se construir, sendo no entanto mais estável e não tão facilmente abalável como a imagem (Walker, 2010).

Pelo exposto pode-se inferir que a reputação corporativa corresponde à avaliação geral de um stakeholder de uma empresa ao longo do tempo, e que ela permitirá garantir uma vantagem competitiva, sendo muitas vezes identificada como uma barreira à entrada de novos concorrentes. Resta levantar uma questão: Como é que os diferentes stakeholders percebem a identidade da empresa e constroem a sua imagem? A resposta está na comunicação corporativa.

\section{A COMUNICAÇÃO CORPORATIVA.}

Gray e Balmer (1998) defendem que a comunicação corporativa é o conjunto agregado de mensagens através das quais a organização leva a sua identidade até aos diferentes stakeholders. Ela apresenta-se como o elo de ligação entre e identidade, e a imagem e reputação da organização, como se apresenta na figura I.

De acordo com o modelo, a comunicação apresenta-se como o elo crítico entre a identidade e imagem traduzindo-se no conjunto de ações com vista a tingir os diferentes públicos. Assim, as fontes comunicativas englobam (Gray \& Balmer, 1998): a nomenclatura, o design gráfico, as declarações formais, a arquitetura, a relação com os meios de comunicação social e as interações rotineiras. 
Figura I

Modelo operacional da comunicação corporativa

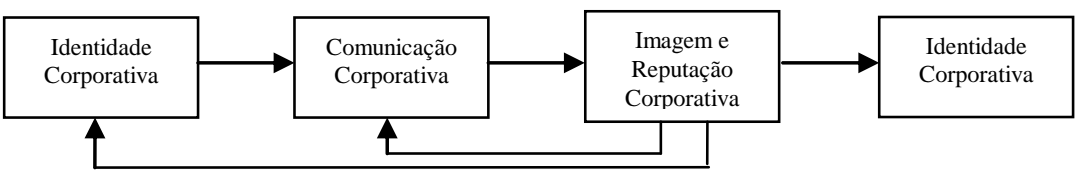

Fonte: Gray e Balmer (1998)

A nomenclatura evidencia o papel do nome identificativo da instituição, das suas divisões e produtos. O design gráfico traduz a identificação visual da organização, que deve ser sempre ajustada à identidade, com a correta articulação entre logótipo e nomenclatura, considerando-se o logótipo o cerne do sistema gráfico, e por essa razão não deve sofrer alterações abruptas que possam conduzir a uma não identificação da empresa, produto ou marca. As declarações formais incluem a missão e os slogans organizacionais, que devem refletir a cultura da empresa. A arquitetura reflete parte da cultura organizacional, pelo seu layout interior, e permite identificação visual, como o design exterior do edifício. Em empresas de serviços esta fonte comunicativa assumirá um papel preponderante, pois o conforto e qualidade do ambiente interno terão um impacto imediato e direto quer nos stakeholders internos quer nos externos.

A relação com os meios de comunicação social tem importância estratégica para o sucesso da projeção da imagem, pois tem um peso significativo na formação da imagem pública da organização. A este propósito, Deephouse (2000) sugere que os meios de comunicação social têm um poder extremo, influenciando a forma como a opinião pública pensa e sente a propósito das temáticas da atualidade, o que se aplica à reputação, pois a divulgação de informação, de mensagens, sobre as organizações é uma constante. Assim, a reputação aparecerá como uma avaliação global da organização apresentada nos meios de comunicação.

Finalmente, as interações rotineiras estão focadas no funcionário, sendo vital que se procure sua constante e continua motivação. Dortok (2006) enfatiza que o funcionário precisa de sentir confiança na organização, e que só através dessa segurança poderá ter o melhor desempenho possível contribuindo para a qualidade da identidade e, por consequência, para a projeção da imagem, pois ele é muitas vezes o grande veículo da imagem organizacional aos olhos dos restantes stakeholders, influenciando a reputação.

O modelo proposto por Gray e Balmer (1998) traduz efetivamente a realidade que envolve o processo de formação da reputação. Numa 
análise cuidada percebe-se que a comunicação corporativa assume um papel preponderante em todo o processo. No entanto, convém não descurar a existência de fatores exógenos que poderão ter um impacto quer na imagem quer na reputação. Veja-se o que acontece em situações de crise: a imagem da organização é a primeira a sentir um abalo e que se não for devidamente trabalhada e de imediato, em função do tipo de crise, poderá colocar em causa a reputação. Simon (2001) aponta que em situações de influência de fatores exógenos, como as situações de crise, logo que a história de torna pública não é possível voltar atrás, pelo que é imperioso que de imediato se faça uma correta gestão da comunicação para minorar os impactes, de modo a que imagem e reputação não saiam severamente afetadas.

Gray e Balmer (1998) acrescentam que os fatores exógenos podem não representar necessariamente uma ameaça, poderão constituir oportunidades de projetar a imagem e reputação organizacional. A título de exemplo apontam a capacidade de controlo de recursos críticos, boas relações de qualidade e mesmo o fator sorte. Para que no decorrer deste processo se garanta a estabilidade da identidade interna da organização é fundamental que se procure efetuar um feedback contínuo sobre o impacte da imagem e reputação na comunicação corporativa e na identidade, pois só assim é possível avaliar a sua correta gestão neste trabalho contínuo.

Para Riel e Balmer (1997), a comunicação corporativa, enquanto elo de ligação entre a identidade e imagem/reputação, pode é o processo pelo qual os stakeholders percebem a identidade, a imagem e reputação da organização.

Pela análise do modelo proposto, é possível afirmar que a reputação é evolutiva ao longo do tempo e é o resultado de interações repetidas e de experiências acumuladas nos relacionamentos com a organização. É um facto que imagens positivas irão gerar uma reputação favorável. Assim, reputação e imagem são conceitos distintos, a imagem apresenta-se como o modo pelo qual os stakeholders vêm a organização, é a sua imagem mental. A reputação requer avaliação e juízos de valor efetuados pelos stakeholders face aos atributos da organização. Gotsi e Wilson (2001) consideram que existe uma relação dinâmica entre ambas, pois entendem que a reputação é a avaliação feita tendo por base as experiências diretas dos stakeholders com a organização, mediadas pela comunicação corporativa, levando-os a efetuar comparações com organizações concorrentes.

É um facto que identidade e imagem se assumem como elementoschave para a conquista de uma boa reputação que conduza a uma 
vantagem competitiva intangível. Resta saber como se pode determinar, ou avaliar, essa reputação.

\section{MEDIR/AVALIAR REPUTAÇÃO CORPORATIVA}

Tal como se verificou anteriormente, a interpretação do conceito de reputação é relativamente ambígua e suscita confusão, o que dificulta a sua avaliação. Para maior segurança das organizações a avaliação da reputação assume um papel determinante no que concerne à identificação de pontos fortes e fracos que terão o seu reflexo na identidade.

De modo a perceber como essa avaliação se pode efetuar procurou-se identificar na literatura instrumentos, escalas ou indicadores que permitam perceber a performance das organizações no que concerne à reputação. Assim, apresentar-se-á um conjunto de modelos ou escalas. Optou-se por começar por escalas centradas em rankings de meios de comunicação social, de seguida mostram-se modelos desenvolvidos por académicos, também focados em stakeholders individuais (escalas de equidade de marca, escalas de imagem, escalas de identidade), e por fim modelos voltados para uma avaliação multistakeholder.

No que concerne a rankings definidos pelos media encontra-se o estudo da Fortune's America Most Admired Companies (AMAC), que procura junto dos diretores executivos das organizações classificar a reputação dos concorrentes, tendo por base o estudo de oito atributos chave: estabilidade financeira, valor dos investimentos de longo prazo, uso dos bens corporativos, capacidade inovadora, qualidade da gestão, qualidade dos produtos e serviços, capacidade para atrair e reter recursos humanos talentosos, reconhecimento em responsabilidade social (Chun, 2005; Schwaiger, 2004). Este ranking, inicialmente, aplicava-se apenas nos Estados Unidos, tendo posteriormente sido alargado a treze países (em 1997), passando a denominar-se Global Most Admired Companies (GMAC) (Schwaiger, 2004).

Ainda nesta linha de avaliação encontra-se o ranking do The Financial Times World's the Most Respected Companies, que procura avaliar a perceção dos diretores executivos concorrentes com base em oito critérios: força e boa aplicabilidade estratégica, maximização da satisfação e lealdade do consumidor, liderança de negócio, qualidade dos produtos e serviços, consistência da performance financeira, força da cultura corporativa, sucesso das mudanças de gestão, globalização do negócio (Chun, 2005). 
Como se verifica, os rankings apresentados têm um grande enfoque em critérios financeiros e estratégicos, estando muito voltados para o estudo da relação entre reputação e outras variáveis financeiras ou estratégicas. Independentemente da a importância do valor financeiro, existem outros fatores que se revestem de suma importância quando se procura avaliar a reputação, como é o caso da qualidade da gestão e da relação com os stakeholders internos, pois existem fatores emocionais que têm impacto na identidade da organização, que por sua vez se reflete na imagem e na reputação. Assim, na tentativa de superar este gap, é necessário desenvolver modelos de avaliação que levem em conta outros fatores, como é o caso das escalas de equidade de marca.

Com base no conceito de equidade de marca começaram a surgir tentativas de medir a reputação, pois a credibilidade corporativa é considerada uma das dimensões da imagem corporativa que pode afetar a equidade de marca, que se encontra diretamente relacionada com a reputação. Nesta linha de pensamento, Caruana e Chircop (2000) consideram que a marca corporativa e a reputação são fatores cruciais para as empresas. Com o intuito de avaliar a reputação através da marca realizaram um estudo numa empresa de bebidas em Malta onde desenvolveram uma escala de reputação de doze itens: qualidade dos produtos produzidos; níveis de publicidade; atividades de patrocínio; abertura para realização de visitas à empresa; empresa com estabilidade, revela cuidados com os funcionários; os funcionários são bem formados/preparados; oferta de produtos bem conhecidos; gestão forte; realiza muita publicidade; é uma empresa muito conhecida; rentabilidade.

Pela análise da escala verifica-se que existe uma maior abrangência de áreas, não se encontrando qualquer foco na área financeira. Não obstante a validade da ferramenta criada, os autores chamam a atenção para a possibilidade de ocorrer efeito de halo, pois os consumidores podem efetuar uma avaliação com base nas perceções prévias que têm, não prestando atenção à realidade do momento. $\mathrm{O}$ facto de a escala ter sido criada para a indústria das bebidas poderá constituir uma limitação à sua aplicabilidade noutras indústrias, e quando aplicada somente a consumidores levará também a uma limitação no que concerne a uma maior validade dos resultados.

Considerando que imagem e reputação estão intrinsecamente relacionadas e que a reputação poderá ser uma variável endógena para avaliar a satisfação e perceção de qualidade, Brown e Dacin (1997) desenvolveram estudos de avaliação de imagem, recorrendo à reputação, com base em Escalas de Imagem. 
Brown e Dacin (1997) realizaram um estudo com o objetivo de avaliar a influência das associações cognitivas que o consumidor faz relativamente a uma organização com base na avaliação dos produtos. No estudo introduziram dois constructos: a habilidade corporativa, que se relaciona com a capacidade da empresa produzir e divulgar os seus outputs, e a responsabilidade social corporativa, que reflete as atividades relacionadas com as obrigações sociais da empresa. Para a concretização do seu objetivo introduziram seis variáveis: a avaliação do produto, a sofisticação do produto, a responsabilidade social do produto, a avaliação corporativa, a habilidade corporativa e a responsabilidade social corporativa.

Estudos deste cariz implicam que a reputação seja avaliada como um conceito multidimensional, o que pode ser complicado, pois o consumidor pode revelar um número limitado de associações para uma dada empresa num dado momento. No entanto, é de ter em consideração que a imagem corporativa é avaliada multidimensionalmente.

LeBranc e Nguyen (1996) identificaram que os seguintes fatores influenciam a imagem organizacional: a identidade corporativa, a reputação (dos diretores), a oferta de serviço, o ambiente físico, o contacto pessoal e o acesso ao serviço. Tendo em consideração estes fatores desenvolveram um estudo na banca de modo a avaliar a imagem corporativa, onde consideraram 36 variáveis de acordo com os fatores em estudo. Os resultados demonstraram que a imagem corporativa está muito dependente do acesso ao serviço e que a reputação dos diretores é um fator que afeta consideravelmente a imagem.

De acordo com os resultados, verifica-se que a reputação acaba por ser avaliada como elemento que tem impacto na imagem. No entanto, de acordo com a escala não é possível avaliar em profundidade a reputação em si.

Para além de escalas de imagem também é possível encontrar na literatura Escalas de Identidade, que procuram avaliar a identidade corporativa e sua relação com a reputação. Balmer e Soenen (1999) desenvolveram um modelo de avaliação da reputação: "The ACID TEST" (Actual, Communicated, Ideal, Desired Identity). Os autores afirmam que ao aplicar o modelo é necessário ter em consideração quatros tipos de identidade: A identidade atual: aquilo que a organização é; a identidade comunicada: como é que a organização é percebida pelos públicos e como comunica; a identidade ideal: a posição ótima da organização no mercado, tendo em consideração as suas forças a habilidades; a identidade desejada: a que os gestores de topo desejam 
alcançar. Para cada tipo de identidade os autores incluem diferentes elementos a avaliar de modo a inferirem conclusões sustentadas.

Com a aplicabilidade do modelo os autores afirmam que é possível determinar fraquezas na estratégia de identidade adotada e formas de mudança para melhorar a identidade atual, levando a uma redefinição da gestão que terá o seu impacto na reputação.

Com se pode constatar, as medidas de avaliação apresentadas previamente estão muito centradas na visão da reputação de um único stakeholder (consumidor, gestor ou funcionário), e avaliam a reputação através de elementos-chave: identidade ou imagem. Considerando que as organizações vivem inseridas num contexto, é importante que a reputação seja avaliada pelo conjunto das perceções dos diferentes stakeholders. Assim, Fombrum, Gardberg e Server (1999), Davies et al. (2003) e Schwaiger (2004), desenvolveram medidas de avaliação da reputação multistakeholder.

Fombrum, Gardberg e Server (1999) desenvolveram o modelo de Quociente de Reputação (Reputation Quotient). Através de vários estágios com estudos piloto e focus group, os autores identificaram um total de vinte itens que organizaram em seis dimensões. Os itens foram gerados a partir de rankings de media (incluindo o ranking AMAC), com a adição de itens relativos a imagem e reputação presentes na literatura. $\mathrm{O}$ modelo proposto pelos autores está organizado e, seis dimensões, tal como se apresenta no quadro III.

Os autores conseguiram conferir uma maior validade ao introduzirem os fatores de apelo emocional e de apelo racional, pois consideram que a reputação é um conceito que deve combinar estes dois fatores (Fombrum, Gardberg \& Server, 1999). Pela observação dos diferentes itens inerentes a cada dimensão, e considerando o número e tipo de dimensões incluídas, o modelo apresenta-se mais robusto e sólido para a avaliação da reputação.

Davies et al. (2003) desenvolveram a Escala de Carácter Corporativo, que procura medir a reputação corporativa do ponto de vista interno e externo, em simultâneo. Através de um estudo com mais de 4600 pessoas, os autores pediam aos participantes para descreverem a avaliarem as organizações como se fossem um ser humano, tendo recorrido a um teste similar ao de avaliação de personalidade. Assim, a escala foi dividida em sete dimensões, com um total de treze facetas distribuídas pelas dimensões, e cinquenta e um itens de classificação (adjetivos) das diferentes facetas. No quadro IV apresentam-se as sete dimensões da escala. 


\section{Quadro III}

Quociente de reputação

\begin{tabular}{|l|l|}
\hline \multicolumn{1}{|c|}{ Itens } & \multicolumn{1}{|c|}{ Dimensões } \\
\hline $\begin{array}{l}\text { Tenho um bom sentimento a propósito da organização } \\
\text { Admiro e respeito a organização } \\
\text { Confio na organização }\end{array}$ & Apelo Emocional \\
\hline $\begin{array}{l}\text { Fica por trás dos seus produtos e serviços } \\
\text { Desenvolve produtos e serviços inovadores } \\
\text { Oferece elevada qualidade de produtos e serviços } \\
\text { Oferece produtos e serviços que têm boa relação de preço }\end{array}$ & Produto e Serviço \\
\hline $\begin{array}{l}\text { Tem excelente capacidade de liderança } \\
\text { Tem uma visão clara do seu futuro } \\
\text { Reconhece e tira vantagem das oportunidades de mercado }\end{array}$ & Visão e Liderança \\
\hline $\begin{array}{l}\text { É bem gerida } \\
\text { Parece ser uma boa empresa para trabalhar } \\
\text { Parece ser uma empresa que teria bons funcionários }\end{array}$ & Ambiente Laboral \\
\hline $\begin{array}{l}\text { Apoia boas causas } \\
\text { É ambientalmente responsável } \\
\text { Mantém um bom nível na forma como trata os seus funcionários }\end{array}$ & $\begin{array}{l}\text { Responsabilidade } \\
\text { Social e Ambiental }\end{array}$ \\
\hline $\begin{array}{l}\text { Tem um bom recorde de rentabilidade } \\
\text { Parece ser um investimento de baixo risco } \\
\text { Tende a ter uma melhor performance que os seus concorrentes } \\
\text { Parece ser uma empresa com forte prospeção de crescimento para } \\
\text { o futuro }\end{array}$ & $\begin{array}{l}\text { Performance } \\
\text { Financeira }\end{array}$ \\
\hline
\end{tabular}

Fonte: Elaborado a partir de Fombrum, Gardberg e Server (1999)

Pela análise da escala percebe-se que a personalidade é usada como ferramenta para aceder à reputação da organização.

Schwaiger (2004) mostrou-se preocupado com a necessidade de se proceder a uma avaliação da reputação atendendo aos diferentes stakeholders, tendo desenvolvido um método de avaliação da reputação. Considerando que esta é bidimensional, ele sugere a divisão em duas dimensões: a componente cognitiva, que denomina de competência, e componente afetiva, que denomina de simpatia.

Quadro IV

Dimensões da Escala de Caráter Corporativo

\begin{tabular}{|l|l|}
\hline \multicolumn{1}{|c|}{ Dimensões } & \multicolumn{1}{c|}{ Facetas } \\
\hline Afabilidade & $\begin{array}{l}\text { Cordialidade } \\
\text { Empatia } \\
\text { Integridade }\end{array}$ \\
\hline Competência & $\begin{array}{l}\text { Consciência } \\
\text { Tecnocracia }\end{array}$ \\
\hline \multirow{2}{*}{ Empresa } & $\begin{array}{l}\text { Modernidade } \\
\text { Aventura } \\
\text { Audácia }\end{array}$ \\
\hline
\end{tabular}




\begin{tabular}{|c|c|}
\hline Elegância & $\begin{array}{l}\text { Elegância } \\
\text { Prestigio } \\
\text { Orgulho }\end{array}$ \\
\hline Crueldade & $\begin{array}{l}\text { Egoísmo } \\
\text { Domínio }\end{array}$ \\
\hline Machismo & \\
\hline Informalidade & \\
\hline
\end{tabular}

Através de um estudo realizado em duas fases, Schwaiger (2004) identifica as seguintes categorias de avaliação da reputação: qualidade dos empregados; qualidade da gestão; performance financeira; qualidade dos produtos e serviços; liderança de mercado; orientação para o cliente; atratividade; responsabilidade social; comportamento ético; confiança; atitude justa relativamente aos concorrentes; transparência e abertura; e credibilidade. $\mathrm{O}$ autor defende que a reputação deve ser medida tendo em consideração um portefólio com as duas dimensões: simpatia e competência.

Após a explanação das diferentes propostas de avaliação da reputação corporativa, parece sensato afirmar que há ainda muito trabalho para desenvolver, pois grande parte das escalas apresentadas estão ajustadas à avaliação da imagem ou identidade, sendo a reputação avaliada de forma implícita. Por outro lado as mediadas de ranking dos media mostram-se muito centradas em espetos financeiros e estratégicos, o que induz para uma maior aceitabilidade do modelo do quociente de reputação de Fombrum, Gardberg e Server (1999), que consideram stakeholders internos e externos, que tem maior abrangência de dimensões.

\section{REPUTAÇÃO CORPORATIVA COMO ANTECEDENTE}

De facto, os modelos de avaliação da reputação corporativa vão muito além da análise da imagem e identidade. Assim, uma vez apresentados as principais modelos, foi decidido apresentar nesta seção as diversas temáticas analisadas na revisão efetuada sobre a reputação corporativa, dado que esta pode ser um meio e não um fim em si.

Minocha (2009), Podnar, Tuškej e Golob (2012), Graig e Brennan (2012) e Ruth e York (2004) exploram a complexidade da linguagem e da informação semântica na reputação cooperativa e concluem que ela é específica do contexto e está relacionada com a fonte da mensagem. Uma questão é clara, questões de semântica podem causar danos na reputação empresarial. 
Relacionado com estes estudos está a questão da gestão de situações críticas/problemáticas. Os estudos de Linthicum, Reitenga e Sánchez (2010), Laufert e Coombs (2006), Claeys, Cauberghe e Vyncke (2010), Hasseldine, Salama e Toms (2005) e Verhoeven et al. (2012) se destacam ao demonstrar que aspetos conflituosos, geradores de situações problemáticas, geram reputação negativa. Como tal, devem ser evitáveis

O estudo das auditorias (Schöndube-Pirchegger, B. \& Schöndube, 2011) é cirúrgico ao apresentar como o comportamento dos stakeholders pode ser moldado após o resultado de auditorias.

Igualmente pertinente para a reputação é o estudo de Fong, Lee e Du (2013), onde se conclui que, independentemente da reputação "transferida", a animosidade anti nipónica manteve-se no mercado. Como tal, a reputação anterior da empresa e dos seus gestores joga um papel importante na construção da própria reputação (Bae \& Cameron, 2006; Truscott, Bartlett \& Tywoniak, 2012), o que indica que a reputação é cumulativa ao longo do tempo.

Um novo tema que tem emergido na literatura é o da responsabilidade social e do seu impacto na reputação (Mutch \& Aitken, 2009; Mitra, 2011; Pomering \& Johnson, 2009; Trustcott et al., 2009; Duhé 2009), onde há uma total coincidência na importância da responsabilidade social na reputação corporativa.

Só um estudo analisa a competitividade/desempenho da empresa, como resultado da sua reputação (Rose \& Thomsen, 2004) tendo concluído que a reputação é importante para o desempenho positivo da empresa.

Finalmente, há estudos que relacionam a qualidade de reputação empresarial e a satisfação (Helm et al 2001; Loureiro \& Kastenholz, 2011; Toms 2002), dando destaque à importância da reputação empresarial na satisfação do consumidor.

\section{CONCLUSÕES, LIMITAÇÕES E ESTUDOS FUTUROS}

As empresas vivem a uma escala global, levando-as a capitalizar todos os ativos na procura de vantagens competitivas. Entre esses ativos encontra-se a reputação corporativa. Na procura de um conceito de reputação é clara a falta de consenso, chegando por vezes a ser associado ou entendido como imagem. No entanto, imagem e reputação são conceitos distintos: a imagem pode ser vista como um dos elementoschave da reputação, assim como a identidade corporativa. 
A identidade assume-se como a realidade da organização, englobando a cultura, os valores e a missão da organização (Chun, 2005; Brady, 2005). Por sua vez, a imagem refere-se à ideia mental que os públicos têm da organização. Finalmente, a reputação diz respeito aos juízos de valor que esses públicos fazem dos atributos da organização (Barnett, Jerminer \& Lafferty, 2006; Gray \& Balmer, 1998).

Se a reputação contribui para a vantagem competitiva das empresas, é importante que se proceda à sua avaliação, recorrendo a escalas ou modelos completos, procurando-se analisar as diferentes variáveis que têm impacte na reputação. Dos variados modelos, o mais completo parece ser o proposto por Fombrum, Gardberg e Server (1999). No entanto, é necessário ter consciência da necessidade de estender esses modelos incluindo a reputação como antecedente da performance, da satisfação, da lealdade e da conquista de mercado.

No que concerne a modelos ou escalas de avaliação de reputação muito ainda há para desenvolver. Claramente, é necessário uma análise aprofundada para se conseguir fazer um levantamento amplo das variáveis e constructos potencialmente importantes.

\section{REFERÊNCIAS BILBIOGRÁFICAS}

Bae, J. e Cameron, G. T. (2006). "Conditioning effect of prior reputation on perception of corporate giving". Public Relations Review, Vol. $32, n^{\circ} 2,144-150$.

Balmer, T. e Soenen, B. (1999). "The ACID test of corporate identity management". Journal of Marketing Management, Vol. 15, 69-92.

Barnett, M., Jerminer, J. e Lafferty B. (2006). "Corporate Reputation: the definitional landscape". Corporate Reputation Review, Vol. 9, $\mathrm{n}^{\circ}$ 1, 26-38.

Brady, A. (2005). The Sustainability Effect - Rethinking Corporate Reputation in the 21st Century. Londres: Palgrave.

Brown, J. e Dacin, A. (1997). "The company and the product: corporate associations and consumer product responses". Journal of Marketing, Vol. 61, $\mathrm{n}^{\circ}$ 1, 244-249.

Caruana, A. (1997). "Corporate reputation: concept and measurement". The Journal of Product and Brand Management, Vol. 6, $\mathrm{n}^{\circ}$ 2, 109118.

Caruana, A. e Chircop (2000). "Measuring corporate reputation: a case example". Corporate Reputation Review. 31(1): 43-57; 
Claeys, A.-S., Cauberghe,V. e Vyncke, P. (2010). "Restoring reputations in times of crisis: An experimental study of the Situational Crisis Communication Theory and the moderating effects of locus of control". Public Relations Review, Vol. 36, n 3, 256-262.

Craig, R. J. e Brennan, N. M. (2012). "An exploration of the relationship between language choice in CEO letters to shareholders and corporate reputation". Accounting Forum, Vol. 36, n 3, 166-177.

Chun, R. (2005). "Corporate reputation: meaning and measurement". International Journal of Management Reviews, Vol. 7, $\mathrm{n}^{\circ}$ 2, 91-109.

Davies, G., Chung, R., Silva, R. e Roper, S. (2003). Corporate Competitiveness. Londres: Routledge.

Deephouse, D. (2000). "Media reputation as a strategic resource: an integration of mass communication and resource-based theories". Journal of Management, Vol. 26, $\mathrm{n}^{\circ}$ 6, 1091-1112.

Dortok, A. (2006). "A Managerial look at the interaction between internal communication and corporate reputation". Corporate Reputation Review, Vol. 8, no 4, 322-338;

Duhé, S. C. (2009). "Good management, sound finances, and social responsibility: Two decades of U.S. corporate insider perspectives on reputation and the bottom line". Public Relations Review, Vol. $35, \mathrm{n}^{\mathrm{o}} 1,77-78$.

Fong, C.-M., Lee, C.-L. e Du, Y. (2013). "Target reputation transferability, consumer animosity, and cross-border acquisition success: A comparison between China and Taiwan". International Business Review, Vol. 22, $\mathrm{n}^{\circ}$ 1, 174-186.

Fombrum, J., Gardberg, A. e Server, M. (1999). "The reputation quotient: a multiple stakeholder measure of corporate reputation". Journal of Brand Management, Vol. 7, $\mathrm{n}^{\circ}$ 4, 241.255.

Gotsi, M. e Wilson, M. (2001). "Corporate Reputation: seeking a definition". Corporate Communications, Vol. 6, n 1, 241-255;

Gray, E. e Balmer, J. (1998). "Managing corporate Image and corporate reputation". Long Range Planning, Vol. 31, no 5, 695-702;

Hasseldine, J., Salama, A. I. e Toms J. S. (2005). "Quantity versus quality: the impact of environmental disclosures on the reputations of UK Plcs". The British Accounting Review, Vol. 37, n 2, 231-248.

Helm, S. Garnefeld, I. e Tolsdorf, J. (2009). "Perceived corporate reputation and consumer satisfaction - An experimental exploration of causal relationships". Australasian Marketing Journal, Vol. 17, $\mathrm{n}^{\circ}$ 2, 69-74. 
Keh, H. e Xie, Y. (2009). "Corporate reputation and customer behavioral intentions: The roles of trust, identification and commitment". Industrial Marketing Management, Vol. 38, $\mathrm{n}^{\circ}$ 7, 732-742.

Laufer, D. e Coombs, W. T. (2006). "How should a company respond to a product harm crisis? The role of corporate reputation and consumer-based cues". Business Horizons, Vol. 49, n 5, 379-385.

LeBranc, G. e Nguyen, N. (1996). "Cues used by customers evaluating corporate image in service firms: an empirical study in financial institutions". International Journal of Service Industry Management, Vol. 7, $n^{\circ}$ 2, 44-56;

Linthicum, C., Reitenga, A. L. e Sánchez, J. M. (2010). "Social responsibility and corporate reputation: The case of the Arthur Andersen Enron audit failure". Journal of Accounting and Public Policy, Vol. 29, n 2, 160-176.

Loureiro, S. e Kastenholz, K. (2011). "Corporate reputation, satisfaction, delight, and loyalty towards rural lodging units in Portugal". International Journal of Hospitality Management, Vol. 30, $\mathrm{n}^{\mathrm{o}} 3$, 575-583.

Minocha, S. (2009). "The language of legitimation: The narrative of reputation management within corporate disclosures". Critical Perspectives on Accounting, Vol. 20, $\mathrm{n}^{\mathrm{o}}$ 2, 289-291.

Mitra, R. (2011). "Framing the corporate responsibility-reputation linkage: The case of Tata Motors in India". Public Relations Review, Vol. 37, no 4, 392-398.

Mutch, N. e Aitken, R. (2009). "Being fair and being seen to be fair: Corporate reputation and CSR partnerships". Australasian Marketing Journal, Vol. 17, n 2 , 92-98.

Podnar, K., Tuškej, U. e Golob, U. (2012). "Mapping semantic meaning of corporate reputation in global economic crisis context: A Slovenian study Original”. Public Relations Review, Vol. 38, n5, 906-915.

Pomering, A. e Johnson, L. W. (2009). "Constructing a corporate social responsibility reputation using corporate image advertising". Australasian Marketing Journal, Vol. 17, $\mathrm{n}^{\mathrm{o}} 2$ 2, 106-114.

Riel, V.; Balmer, T. (1997). "Corporate identity". European Journal of Marketing, Vol. 31, $\mathrm{n}^{\circ}$ 5/6, 340-355.

Rose, C. e Thomsen, S. (2004). "The Impact of Corporate Reputation on Performance: Some Danish Evidence". European Management Journal, Vol. 22, nº 2, 201-210. 
Ruth, J. A. e York, A. (2004). "Framing information to enhance corporate reputation: The impact of message source, information type, and reference point”. Journal of Business Research, Vol. 57, n 1, 14-20.

Schöndube-Pirchegger, B. e Schöndube, J. R. (2011). "Reputation concerns and herd behavior of audit committees - A corporate governance problem". Journal of Accounting and Public Policy, Vol. 30, no 4, 327-347.

Schwaiger, M. (2004). "Components and parameters of corporate reputation - an empirical study". Schmalenbach Business Review. 56: 46-71.

Simon, T. (2001). Defending your Reputation. London: Hawksmere Publishing.

Smaiziene, I. e Jucevicius, R. (2009). "Corporate Reputation: Multidisciplinary Richness and Search for Relevant Definition". Engineering Economics, Vol. 62, nº 2, 91-100.

Toms, J. S. (2002) "Firm resources, quality signals and the determinants of corporate environmental reputation: some UK evidence". The British Accounting Review, Vol. 34, n 3, 257-282.

Truscott, R. A., Bartlett, J. L. e Tywoniak, S. A. (2009). "The reputation of the corporate social responsibility industry in Australia". Australasian Marketing Journal, Vol. 17, nº 2, 84-91.

Turk, J., Jin, Y., Stewart, S. Kim, J. e Hipple. J. R. (2012). "Examining the interplay of an organization's prior reputation, CEO's visibility, and immediate response to a crisis". Public Relations Review, Vol. $38, n^{\circ} 4,574-583$.

Verhoeven, J., Van Hoof, J. J., Keurs, H. e Van Vuuren, M. (2012). "Effects of apologies and crisis responsibility on corporate and spokesperson reputation". Public Relations Review, Vol. 38, n 3, 501-504.

Walker, K. (2010). "A systematic review of the corporate reputation literature: definition, measurement, and theory". Corporate Reputation Review, Vol. 12, nº4, 357-387. 